\title{
Fluxograma de atendimento a crianças e adolescentes em unidade de atenção secundária em saúde mental, durante a pandemia por COVID-19, no Distrito Federal
}

\author{
Elisângela Alves dos Santos ${ }^{[1]}$ \\ Camila Rodrigues Oliveira ${ }^{[2]}$ \\ Marianne Gonçalves de Oliveira ${ }^{[3]}$ \\ Samuel João da Silva ${ }^{[4]}$ \\ Gracielle Pollyanna Mertens Carvalho ${ }^{[5]}$ \\ Ana Paula Oliveira Reis Tuyama ${ }^{[6]}$ \\ Ana Cristina Ferreira dos Reis Almeida ${ }^{[7]}$ \\ Gabriela Lopes da Silva Lustosa ${ }^{[8]}$
}

RESUMO: Este estudo tem o objetivo de definir o fluxo de atendimento a crianças e adolescentes que apresentem sintomas suspeitos de COVID-19 em uma unidade de atenção secundária em saúde mental infantojuvenil, com o intuito de atuar na identificação, notificação e manejo oportuno de casos suspeitos e encaminhamento do usuário para unidade de referência. Trata-se de uma abordagem qualitativa com análise documental, que contribuiu para a criação do fluxograma de atendimento a pacientes suspeitos da COVID-19 no serviço e partiu da indagação de como dinamizar os atendimentos e evitar que os profissionais se coloquem em risco diante a possíveis casos de COVID-19.

Palavras-Chave: Fluxograma, Coronavírus, Análise Documental.

[1] Enfermeira residente em saúde mental infanto-juvenil. Escola Superior de Ciências da SaúdeESCS/FEPECS.

[2] Assistente social residente em saúde mental infanto-juvenil. Escola Superior de Ciências da SaúdeESCS/FEPECS.

[3] Fisioterapeuta residente em saúde mental infanto-juvenil. Escola Superior de Ciências da SaúdeESCS/FEPECS.

[4] Psicólogo residente em saúde mental infanto-juvenil. Escola Superior de Ciências da SaúdeESCS/FEPECS.

[5] Enfermeira Secretaria de Estado de Saúde do Distrito Federal.

[6] Médica Pediatra Secretaria de Estado de Saúde do Distrito Federal.

[7] Fisioterapeuta Secretaria de Estado de Saúde do Distrito Federal.

[8] Enfermeira preceptora em saúde mental infanto-juvenil, mestra em Ciências e Tecnologias em Saúde PPGCTS FCE/UnB e servidora na Secretaria de Estado de Saúde do Distrito Federal. 


\title{
Flowchart of care for children and teenagers in a secondary mental health care unit during the COVID-19 pandemic in the Federal District
}

\begin{abstract}
This study aims to define the flow of care for users who have suspected symptoms of COVID-19 in a secondary care unit in children and teenagers mental health. To act in the identification, notification and timely management of suspected cases and referral of the user to the reference unit. This is a qualitative approach with documentary analysis, which contributed to the creation of the flowchart of care for patients with suspected cases of COVID-19 in the service and started with the question of how to streamline care and prevent professionals from putting themselves at risk in the face of possible cases of COVID-19.
\end{abstract}

Keywords: Flowchart, Coronavirus, Document Analysis.

\section{INTRODUÇÃO}

Esse artigo trata da atuação de uma unidade de atendimento da atenção secundária em saúde mental infanto-juvenil no Distrito Federal, durante o contexto de pandemia pela COVID-19. Esse tema é de extrema relevância, visto que visa dinamizar os atendimentos em saúde mental e evitar o risco de contaminação dos profissionais de saúde e orientar de forma adequada os usuários do serviço. Para isso, se faz necessária uma abordagem crítica e reflexiva sobre o processo de pandemia atual, e de como isso impacta na atuação deste serviço de saúde.

O primeiro caso de contaminação pelo Coronavírus foi relatado na província de Wuhan, na China, no final de dezembro de 2019. O vírus se alastrou rapidamente por vários países. No dia 30 de janeiro de 2020, a Organização Mundial da Saúde (OMS) declarou o surto como Emergência de Saúde Pública de Importância Internacional (ESPII). Dentre as questões apresentadas pelo Comitê de Emergência do Regulamento Sanitário Internacional sobre o Coronavírus (2019 n-Cov), está a preocupação do potencial do vírus se espalhar por países com sistemas de saúde mais fracos e mal preparados para lidar com essas situações ${ }^{1}$. 
Uma vez contaminado, o sujeito pode apresentar quadros leves ou assintomáticos, como também pode sofrer complicações respiratórias que podem ocasionar a morte. Além disso, a transmissão ocorre facilmente, inclusive pelo contato entre mãos. Dentre os sintomas apresentados como leves a moderados se destacam: febre, tosse, cansaço e dispneia. Os casos mais graves, habitualmente estão associados a outras comorbidades. Podem demandar internação com necessidade de oxigenioterapia, e com o agravamento do quadro desenvolver a Síndrome da Angústia Respiratória Aguda, em que se faz necessário tratamento intensivo ${ }^{1}$.

Devido à alta disseminação do vírus, em vários países têm adotado medidas de isolamento social e quarentena. A recomendação é que grupos considerados de risco, como idosos e pessoas com comorbidades, fiquem em casa. $\mathrm{O}$ fechamento de estabelecimentos públicos e privados também foi uma medida adotada para evitar a aglomeração de pessoas ${ }^{1}$.

Observa-se que mesmo com orientações e planos de contingências, os casos de contaminação aumentaram drasticamente. Países como Itália, Espanha e China foram os mais afetados pelos efeitos da pandemia, até o momento. Cada país, com suas particularidades, desenvolveu medidas para amenizar os impactos e os números de mortes. Atualmente os Estados Unidos são considerados o novo epicentro da doença no mundo. Isso se deu em decorrência da demora para implementar ações de isolamento ${ }^{1}$.

A pandemia por COVID-19 representa um dos maiores desafios sanitários em escala mundial desse século. Na primeira semana do mês de abril, poucos meses depois do início da epidemia na China, em dezembro de 2019, já foram reportados mais de 1,5 milhão de casos e 85 mil mortes no mundo, e espera-se que um número ainda maior de casos e óbitos venha a ocorrer nos próximos 
meses. No Brasil, até o dia oito de abril de 2020, foram registrados 15.927 casos confirmados e 800 mortes pela Covid-19²,p.1

No Brasil, o primeiro caso confirmado ocorreu no dia 27 de janeiro, no Estado São Paulo, sendo também o primeiro caso na América do Sul. Nesse momento, o Brasil foi inserido no nível de emergência 2, partindo de "sem casos suspeitos" para "risco iminente". A Portaria n 188, de 3 de fevereiro de 2020, do Ministério da Saúde, decretou Emergência em Saúde Pública de Importância Nacional (ESPIN) em decorrência da Infecção Humana pelo novo Coronavírus (2019-nCov). No dia 6 de fevereiro, declarou a Lei da Quarentena, lei no $13.979^{3}$.

Atualmente, observa-se processo de transmissão comunitária em todo país, tendo até a noite do dia 23 de abril a confirmação de 49.492 casos e 3.313 óbitos. Os dados são preocupantes, visto que são mais de mil mortes (1.172) nos últimos 7 dias $^{4}$. Pelo menos 6.512 pacientes se recuperaram da COVID-19, segundo dados apresentados ao G1 pelas Secretarias Estaduais de Saúde. A maior parte das mortes se concentra nas cidades de: São Paulo, Rio de Janeiro, Fortaleza, Manaus e Recife ${ }^{4}$.

No Distrito Federal, existem 968 casos confirmados, com um total de 25 óbitos. No entanto, os dados não correspondem exatamente à realidade, pois há um considerável índice de subnotificações. O preocupante dessa situação, além das subnotificações e da insuficiência de mecanismos para a realização de testes, se dá pela flexibilização das medidas de distanciamento social, com a reabertura de alguns estabelecimentos comerciais, atrelada à necessidade de a população transitar pela cidade para ir aos seus respectivos postos de trabalho ${ }^{4}$.

Para subsidiar as famílias nesse cenário de extrema desigualdade social e vulnerabilidade socioeconômica, foi aprovada a criação de um auxílio para o pagamento 
de renda básica emergencial, executado pela Caixa Econômica Federal. Por meio da Lei 13.982, de 02 de abril de 2020, milhões de brasileiros que se enquadrarem nos critérios: ser trabalhador maior de dezoito anos, sem emprego formal ativo, renda per capita inferior a meio salário mínimo, não ser beneficiário de outros programas (exceto Bolsa Família), estar registrado no Cadastro Único, entre outros critérios, poderão acessar o valor de seiscentos reais, durante 3 meses.

Dentre as muitas questões éticas surgidas com a pandemia COVID-19, uma, em particular, guarda direta relação com o tema da justiça social enquanto foco investigativo do próprio nexo entre moral e política. Trata-se de sabermos em que medida cabe à sociedade o dever de assegurar aos seus membros uma renda básica, capaz de lhes garantir assim um item elementar de sobrevivência em contraponto aos abusos do poder econômico. Em tais circunstâncias, o debate costuma girar em torno do papel redistributivo do Estado, cuja força reivindicativa coincide normativamente com o pressuposto de um pacto de solidariedade celebrado entre os cidadãos $^{6, p .1}$.

A emergência internacional relacionada à COVID-19, declarada em 30 de janeiro de 2020, pela Organização Mundial da Saúde (OMS), fez com que o foco da comunidade científica se volte para a saúde global. E que as comunidades de saúde busquem estratégias para lidar com as consequências da crise que afetam não apenas a saúde física, como também o bem estar dos indivíduos e seus ambientes de lazer e trabalho.

Sendo o conceito de saúde pela OMS definido como "bem-estar físico, mental e social", entende-se a necessidade de dados e pesquisas permanentes levando-se em consideração não apenas a evolução das doenças específicas, mas também os impactos das crises desencadeadas da pandemia sobre a saúde da população, além de investigações nas áreas sociais, ambientais, econômicas e nas políticas de saúde. Assim, essa pesquisa 
sobre o impacto nas ações em ambiente de trabalho em Saúde Mental Infantojuvenil tornou-se o foco deste trabalho.

O objetivo deste trabalho é, por meio do levantamento de documentos, utilizá-los como instrumentos para subsidiar a construção de fluxo de atendimento aos casos leves de COVID-19. Consideram-se, nesse sentido, os direcionamentos necessários na rede de serviços de saúde da Secretaria de Estado de Saúde do Distrito Federal.

\section{METODOLOGIA}

Trata-se de estudo qualitativo com utilização da análise documental. Diferenciase da revisão bibliográfica, pela natureza dos documentos (o tratamento científico) que são fonte de dados para a pesquisa, podendo ser selecionados: filmes, atas, documentos gerais com jargões, linguagem informal, portanto, qualquer prova que sirva de comprovação ou testemunho. Descreve os elementos que devem ser observados na pesquisa $^{7}$ como: o conteúdo abordado, autores, a relevância, segurança das informações, origem do texto e conceitos-chave.

Tal análise precisa mostrar a localização, identificação, organização e avaliação das informações contidas no documento, além da contextualização dos fatos em determinados momentos. Neste trabalho, optamos por fazer levantamento cronológico das portarias e circulares divulgados por diferentes setores da Secretaria de Estado de Saúde do Distrito Federal (SES DF) nos meses de março e abril de 2020, que serviram de comunicação entre diferentes setores da Secretaria de Saúde e no serviço em que a pesquisa foi realizada, ancoraram a construção do fluxo e a organização de espaço de atendimento aos casos de COVID-19. Além dos documentos divulgados pela SES DF, 
outras notas técnicas escritas pelo Ministério da Saúde, Agência Nacional de Vigilância Sanitária (ANVISA), foram analisadas, conforme registro no Quadro $1^{7-10}$.

Importante destacar que a constante iminência de convocação para atuação na linha de frente aos pacientes acometidos pela doença causada pelo COVID-19 trouxe importantes impactos aos processos de trabalho do serviço, como alteração do volume de atendimentos individuais, interrupção temporária dos atendimentos em grupos terapêuticos ${ }^{7,8}$. As Residências Multiprofissionais nos diversos serviços de saúde são importantes para a contribuição com os diferentes processos de trabalho em saúde e com a alteração do cotidiano deles. Especificamente no caso deste estudo, a Residência Multiprofissional em Saúde Mental Infantojuvenil se dispôs a contribuir com a garantia do atendimento aos casos leves e moderados de sofrimento psíquico em ambulatório de saúde especializado em atender adolescentes e suas famílias.

Quadro 1- Documentos base para construção do fluxograma de atendimento, Brasília, 2020.

\begin{tabular}{|c|c|c|c|}
\hline Título & Data & Endereçamento & $\begin{array}{c}\text { Caracterização do } \\
\text { conteúdo }\end{array}$ \\
\hline $\begin{array}{c}\text { Memorando no29/2020- } \\
\text { SES/SAIS/COASIS/DISSAM }\end{array}$ & $17 / 03 / 2020$ & $\begin{array}{c}\text { Aos gestores dos } \\
\text { serviços } \\
\text { especializados em } \\
\text { Saúde Mental }\end{array}$ & $\begin{array}{c}\text { Esclarece sobre a } \\
\text { importância da } \\
\text { manutenção do } \\
\text { atendimento } \\
\text { psicossocial às } \\
\text { pessoas com } \\
\text { condições } \\
\text { preexistentes de } \\
\text { saúde mental }\end{array}$ \\
\hline
\end{tabular}




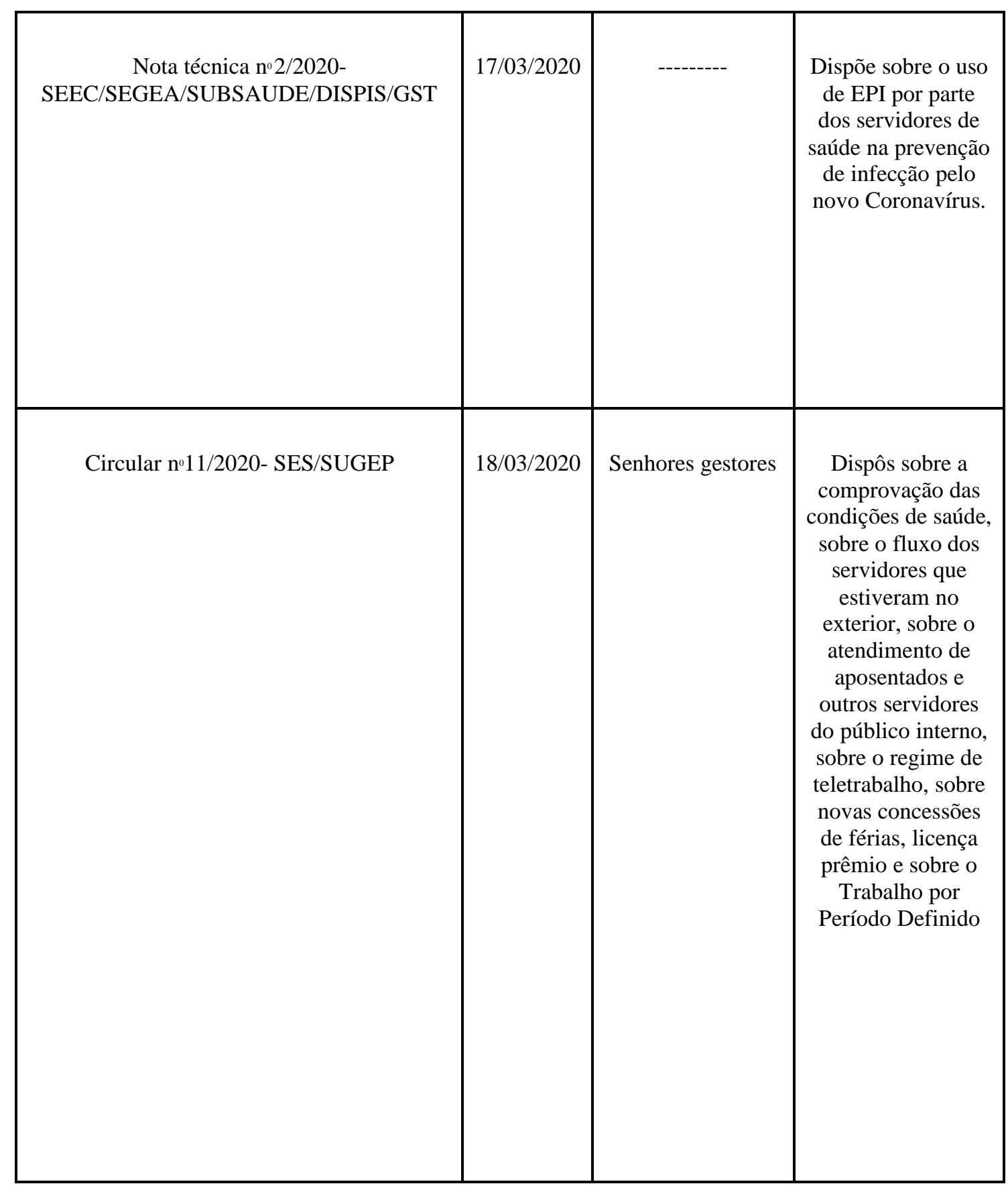




\begin{tabular}{|c|c|c|c|}
\hline Circular no 17/2020- SES/SAA & $18 / 03 / 2020$ & Prezados senhores & $\begin{array}{c}\text { É urgente. Cancela } \\
\text { a vigência do Plano } \\
\text { de Contingência } 5 \text { e } \\
\text { reestipula o } 4 . \\
\text { Coloca a } \\
\text { manutenção dos } \\
\text { serviços } \\
\text { ambulatoriais e dos } \\
\text { procedimentos } \\
\text { cirúrgicos. }\end{array}$ \\
\hline Circular n 15/2020-SES/SUGEP & $20 / 03 / 2020$ & Senhores gestores & $\begin{array}{l}\text { Cancelou as férias, } \\
\text { licença-prêmio, } \\
\text { dispensa de ponto } \\
\text { para congressos e } \\
\text { eventos em geral e } \\
\text { abonos, sendo } \\
\text { marcados e não } \\
\text { iniciados até a data } \\
\text { do documento }\end{array}$ \\
\hline $\begin{array}{l}\text { Despacho -SES/SAIS/COASIS/DIENF } \\
\text { (memorando) }\end{array}$ & $31 / 03 / 2020$ & $\begin{array}{c}\text { GESSAM } \\
\text { Senhora gerente }\end{array}$ & $\begin{array}{l}\text { Memorando que } \\
\text { orienta a triagem } \\
\text { pela equipe de } \\
\text { enfermagem em } \\
\text { epidemia da doença } \\
\text { por Coronavírus. } \\
\text { Dispõe de } \\
\text { fluxograma de } \\
\text { triagem por equipe } \\
\text { de enfermagem em } \\
\text { epidemia pelo } \\
\text { coronavírus em } \\
\text { serviço de saúde } \\
\text { mental. }\end{array}$ \\
\hline
\end{tabular}




\begin{tabular}{|c|c|c|c|}
\hline $\begin{array}{c}\text { Nota informativa A saúde mental e a } \\
\text { Pandemia de COVID 19- impactos e } \\
\text { orientações para profissionais de saúde- } \\
\text { DISSAM }\end{array}$ & $23 / 04 / 2020$ & Gerente & $\begin{array}{c}\text { Dispõe de cuidados } \\
\text { para a saúde mental } \\
\text { dos profissionais de } \\
\text { saúde em meio à } \\
\text { pandemia COVID- } \\
19\end{array}$ \\
\hline $\begin{array}{l}\text { Memorando n 40/2020- } \\
\text { SES/SAAIS/COASIS/DISSAM/GENASAM }\end{array}$ & $26 / 03 / 2020$ & $\begin{array}{c}\text { Aos CAPS, } \\
\text { COMPP, } \\
\text { Adolescentro } \\
\text { Senhores gestores }\end{array}$ & $\begin{array}{l}\text { Considera o serviço } \\
\text { de saúde mental } \\
\text { essencial para os } \\
\text { indivíduos. Orienta } \\
\text { a implementação da } \\
\text { Triagem por } \\
\text { Equipe de } \\
\text { Enfermagem em } \\
\text { Epidemia da } \\
\text { Doença pelo } \\
\text { Coronavírus } \\
\text { segundo público } \\
\text { alvo, local, } \\
\text { materiais, atuação e } \\
\text { fluxo do Plano de } \\
\text { Contingência }\end{array}$ \\
\hline Circular 2/2020- SES/SULOG/DLOG & $01 / 04 / 2020$ & $\begin{array}{l}\text { Às unidades } \\
\text { hospitalares, às } \\
\text { superintendências } \\
\text { regionais de saúde, } \\
\text { aos núcleos de } \\
\text { logística } \\
\text { farmacêtica, ao } \\
\text { CRDF/SAMU }\end{array}$ & $\begin{array}{l}\text { Distribuição de EPI } \\
\text { pelo almoxarifado } \\
\text { Central, } \\
\text { organizando quais } \\
\text { unidades de saúde e } \\
\text { em quais dias } \\
\text { deveria proceder o } \\
\text { recebimento dos } \\
\text { documentos. }\end{array}$ \\
\hline
\end{tabular}




\begin{tabular}{|c|c|c|c|}
\hline $\begin{array}{c}\text { Circular n 6/2020- } \\
\text { FEPECS/CPEX/GREEX/NR/COREMU }\end{array}$ & 24/04/2020 & $\begin{array}{c}\text { Preceptores, } \\
\text { tutores e } \\
\text { coordenadores dos } \\
\text { Programas de } \\
\text { Residência em } \\
\text { Áreas } \\
\text { Profissionais e } \\
\text { Multiprofissionais } \\
\text { de Saúde da } \\
\text { Secretaria de } \\
\text { Estado do Distrito } \\
\text { Federal }\end{array}$ & $\begin{array}{c}\text { Orientações sobre } \\
\text { grupo de risco, } \\
\text { rodízio, } \\
\text { afastamentos, } \\
\text { atestados e } \\
\text { teletrabalho o } \\
\text { COVID-19 e as } \\
\text { atividades das } \\
\text { Residências em } \\
\text { Áreas profissionais } \\
\text { e } \\
\\
\end{array} \quad \begin{array}{c}\text { Multiprofissionais } \\
\text { de Saúde da } \\
\text { Secretaria de } \\
\text { Estado do Distrito } \\
\text { Federal }\end{array}$ \\
\hline
\end{tabular}

Fonte: dados da pesquisa, 2020.

A pesquisa foi realizada no Adolescentro, unidade da rede de atenção secundária de saúde mental infanto-juvenil que presta atendimento individual e em grupo para adolescentes de 12 a 18 anos de idade, atende nas modalidades biopsicossocial; presta atenção a adolescentes que viveram violência sexual, além de assistência e tratamento em demandas específicas de transtorno mentais. No atendimento individual oferece aos adolescentes, que já sejam acompanhados no serviço, o atendimento ambulatorial nas especialidades de Psicologia, Neurologia, Psiquiatria, Ginecologia, Terapia Ocupacional, Enfermagem, Serviço Social, Nutrição, Fisioterapia, Fonoaudiologia, Homeopatia, Odontologia e Práticas Integrativas em Saúde (Reiki, Yoga).

Por fim, por se tratar de estudo em que houve análise de documentos oficiais, não há necessidade de aprovação em Comitê de Ética em Pesquisa.

\section{RESULTADOS}




\section{Fluxograma de atendimento aos pacientes no Adolescentro, Brasília, 2020.}

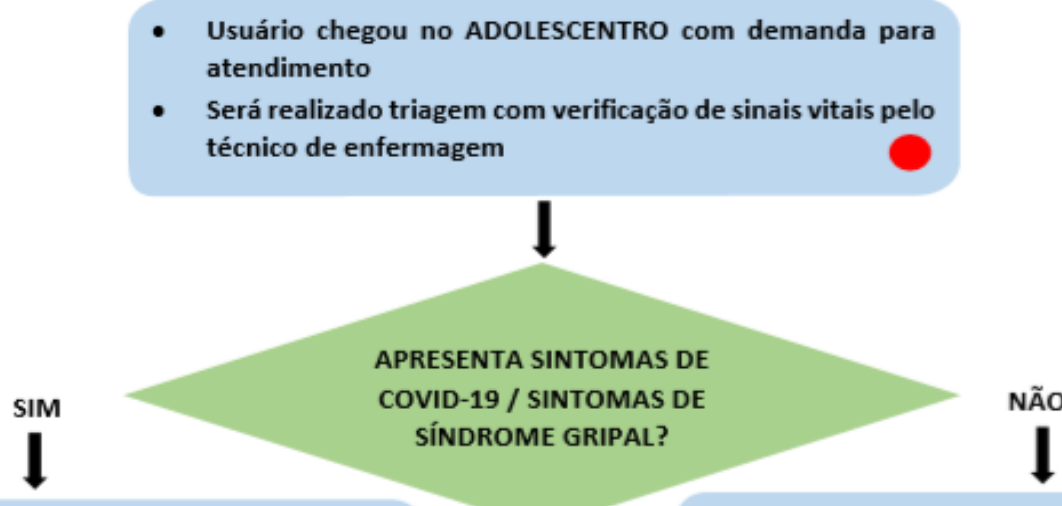

- Colocar uma máscara no indivíduo

- Conduzir para sala específica (consultório 16), visando o isolamento. Enquanto aguarda anamnese específica (Enfermeiro/ Médico).
- Fazer orientação sobre o COVID-19 e seguir com o atendimento normal no ADOLESCENTRO.

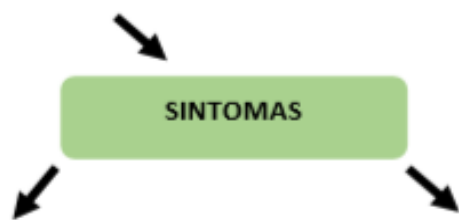

LEVE:

(Tosse, febre, coriza)

\section{Grave:}

(Taquipnéia, dispneia, taquicardia, prostração extrema)

- Orientações gerais sobre o COVID -19, repouso hidratação, alimentação adequada, analgésicos, antitérmicos

- Isolamento domiciliar por 14 dias a contar da data de início dos sintomas.

- Prescrever OSELTAMIVIR para pessoas com condições de risco para complicações.

- Orientar que em caso de piora dos sintomas com evolução para dificuldade respiratória, taquicardia e prostração leve, procurar serviço de emergência (UPAS e Hospitais de referência).

- Após orientação, Apresentar o termo de consentimento livre e esclarecido (TCLE) especifico para o isolamento social com coleta de sua assinatura.

- Comunicar para a UBS que assiste a área de residência do usuário via SEI.

\section{LEGENDA UTILIZAÇ̃̃O DE EPI'S}

Protetor facial ou b́culos de proteçăo, gorro, máscara cirüreica, capote, luvas de procedimento e higienizaçăo frequente das măos com produto alcoólico a $70 \%$ ou com agua e sabonete liquido.

Manter a distância minima de 1 metro, máscara cirúrgica e higienização frequente das măos com produto alcoólico a $70 \%$ ou com àgua e sabonete liquido.
- Realizar transporte sanitário imediato do paciente de ambulância a unidade hospitalar de referência (HRAN).

- Caso não haja possibilidade de transporte próprio da unidade de saúde, a sequência sugerida de acionamento o CORPO DE BOMBEIROS (193) e SAMU (192).

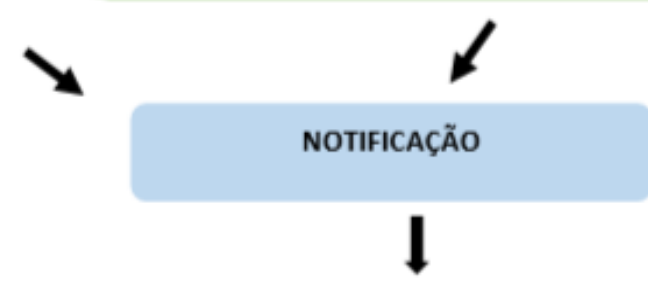

É mandatória a notificação imediata dos casos tratados como suspeitos. O profissional responsável pelo atendimento deve preencher o formulário e a administração será responsável pelo cadastro da notificação via plataforma E-SUS. 
Destaca-se que o produto foi apresentado em reunião de equipe para todos os servidores, que contribuíram criticamente para a melhora do fluxograma produzido pelos residentes, sugerindo alterações como o acréscimo dos Equipamentos de Proteção Individual necessários em cada etapa do atendimento.

A conduta inicial do fluxograma corresponde ao público com demanda para atendimento no Adolescentro, sendo os adolescentes e responsáveis.

Ao chegar no serviço, os usuários serão triados por um técnico de enfermagem, e serão observados quanto aos sintomas de síndrome gripal/COVID-19. Quando o usuário não apresentar sintomas, segue com o atendimento normal. Porém se apresentar sintomas, deverá ser entregue máscara e o paciente segue para uma sala específica visando o isolamento enquanto aguarda anamnese específica realizada por enfermeiro e/ou médico $^{1,10}$.

Os casos são divididos em leves e graves. Nos casos leves estão os indivíduos que apresentam tosse, febre aferida ou relatada de início súbito, coriza, dor de garganta ou dificuldade respiratória, na ausência de outro diagnóstico que tem como sintomas esses relatados. E para os sintomas graves estão: taquipneia, dispneia, taquicardia e prostração extrema $^{1,10}$.

Os leves devem ser manejados com medidas farmacológicas e nãofarmacológicas como repouso, hidratação, boa alimentação, o uso de analgésicos e antitérmicos que serão prescritos pelo médico responsável pelo atendimento. Recomendase isolamento domiciliar por 14 dias a contar da data de início dos sintomas ${ }^{1,10}$.

Indica-se o uso de Oseltamivir nos casos de síndrome gripal com fatores de risco para complicações como: gestantes, puérperas, idosos, crianças menores de 5 anos, 
indígenas com dificuldade de acesso ao serviço de saúde, menores de 19 anos de idade em uso prolongado de ácido acetilsalicílico, doenças hematológicas, anemia falciforme, diabetes mellitus, transtornos neurológicos e do desenvolvimento como disfunção cognitiva, lesão medular, epilepsia, paralisia cerebral, síndrome de Down, pneumopatias, asma, tuberculose, cardiopatias, nefropatias, hepatopatias, acidente vascular encefálico, imunossupressão, neoplasias, HIV/AIDS e obesidade ${ }^{1}$.

A vigilância continuada desses pacientes que estão recebendo acompanhamento ambulatorial é a principal ferramenta para o manejo adequado para esses casos.

Caso haja piora dos sintomas evoluindo para dispneia, taquicardia e prostração leve, orientar para que o usuário procure o serviço de emergência (Unidades de ProntoAtendimentos e hospitais de referência). Após orientação relacionada à COVID-19, apresentar o termo de consentimento livre e esclarecido (TCLE) específico para o isolamento social com coleta de sua assinatura e comunicar para a UBS que assiste a área de residência do usuário, via $\mathrm{SEI}^{10}$.

Para os casos graves, a conduta inclui a estabilização clínica, encaminhamento e transporte a centros de referência ou serviço de urgência e emergência ou hospitalares. Caso não seja possível o transporte próprio da unidade de saúde, a sequência específica para acionamento é o Corpo de Bombeiros (193) e Serviço de Atendimento Móvel de Urgência- SAMU (192) ${ }^{1}$.

A prevenção é essencial para evitar exposição ao vírus, ainda não existe vacina para a COVID-19, para isso é necessário usar EPIs e higienizar corretamente as mãos. Além disso, a máscara deve estar apropriadamente ajustada à face para garantir sua eficácia e reduzir o risco de transmissão. Todos os profissionais devem ser orientados sobre como usar, remover, descartá-las e quanto a higiene das mãos antes e após o uso ${ }^{1}$. 
A notificação imediata dos casos se faz necessária, bem como os suspeitos. E para sintomas leves e graves, o profissional responsável pelo atendimento deve preencher o formulário e o administrativo será responsável pelo cadastro da notificação via plataforma E-SUS ${ }^{10}$.

\section{DISCUSSÃO}

A elaboração de um fluxo de atendimento no serviço secundário de Saúde Mental Infanto-Juvenil é de fundamental importância, considerando a segurança dos pacientes. Tendo em vista a diversidade e a quantidade de informações contidas nos documentos oficiais, é possível demonstrar a dificuldade de organização e a insegurança diante desse cenário, que passa a ser desafiador e desconhecido. Desse modo, o fluxograma de atendimento passa a ser reconhecido como uma ferramenta de trabalho essencial para minimizar o risco de propagação da doença para a população e para os profissionais de saúde.

Os usuários de saúde mental infanto-juvenil, como exemplos deficientes intelectuais, ao virem ao serviço acompanhados de suas respectivas famílias, terão a possibilidade de esclarecimentos em relação à gravidade da situação e os cuidados necessários para evitar a propagação do vírus. Considera-se, por sua vez, que eles encontrem dificuldades ou não teriam a possibilidade de esclarecimentos em outros setores.

A atenção em saúde voltada para saúde mental de crianças e adolescentes e a criação de políticas públicas sobre esse assunto é recente e tem sido considerada um dos desafios da Reforma Psiquiátrica Brasileira. Esse movimento ligado com a construção do SUS, tem como o objetivo o reconhecimento da criança e do adolescente como sujeitos de direitos e responsabilidades ${ }^{11}$. 
Sugerem nesse contexto que os cuidados em saúde mental infanto-juvenil se desenvolvam em diferentes serviços de saúde, como: Unidade Básica de Saúde, Estratégia Saúde da Família (ESF), Centros de Atenção Psicossocial e ambulatórios e hospitais gerais que articulados a uma rede têm como meta a inclusão desses usuários ${ }^{11}$.

A pandemia de COVID-19, no sentido de seu crescimento e, impacto geral na população e nos serviços de saúde, entra num contexto de acesso a muita informação em curto tempo, causando assim impactos principalmente na saúde mental da população e profissionais de saúde, onde todos reagem de maneira diferente a situações estressoras. ${ }^{12}$ Esse impacto durante a pandemia pode ter consequência nas comunidades e nas famílias. Como por exemplo o medo de ficar doente e morrer, pessoas que têm transtornos mentais relacionados principalmente ao distanciamento social, quarentena, isolamento, e a redução de estímulos, preocupação com remédios, alterações do sono, falta de concentração nas tarefas diárias, risco de deterioração de doenças clínicas e de transtornos mentais prévios, risco de adoecimento de profissionais de saúde, o medo e a ansiedade estão entre esses impactos ${ }^{12}$. A não descontinuidade do cuidado a esses pacientes por meio da manutenção dos serviços de saúde mental de modo geral, somada à possibilidade de avaliação de casos suspeitos garantem à população acesso ao atendimento integral no contexto da pandemia.

A limitação do estudo consiste na dificuldade de embasamento de experiências organizadas de atendimento em outros serviços. Dessa forma, essa limitação também representa a contribuição deste estudo.

\section{CONCLUSÃO}

Para uma melhor organização e manejo a usuários com suspeita de coronavírus deve se levar em consideração não só o usuário que busca atendimento, mas também o 
responsável e o profissional que participa do cuidado. Uma vez que o atendimento deve ser prestado de modo a evitar a transmissão. A saúde mental atua como um pilar positivo na aceitação em um momento de isolamento social com muitas mudanças. Alguns impactos infelizmente podem causar consequências tanto na saúde mental quanto na física.

O fluxograma é uma ferramenta de apoio para que esse cuidado seja organizado e bem estruturado por todos os profissionais. Trazendo mais segurança e tranquilidade no atendimento. Nesse contexto, a organização faz toda diferença, assim a identificação dos sintomas, o manejo, os encaminhamentos e a notificação serão executados da melhor forma.

\section{REFERÊNCIAS BIBLIOGRÁFICAS}

1. Ministério da Saúde. Protocolo De Manejo Clínico Do Coronavírus (Covid-19) Na Atenção Primária À Saúde. Brasília - DF, Abril 2020. Organização Mundial Da Saúde (OMS). Constituição. Genebra: OMS, 1948. Disponível em: https://portalarquivos.saude.gov.br/images/pdf/2020/April/08/20200408-

2. Bastos, Leonardo Soares et al. COVID-19 e hospitalizações por SRAG no Brasil: uma comparação até a $12^{\mathrm{a}}$ semana epidemiológica de 2020. Cad. Saúde Pública, Rio de Janeiro, v. 36, n. 4, e00070120, 2020. Disponível em: http://www.scielo.br/scielo.php?script=sci_arttext\&pid=S0102311X2020000406001\&lng=en\&nrm=iso

3. Croda, Julio et al. COVID-19 in Brazil: advantages of a socialized unified health system and preparation to contain cases. Rev. Soc. Bras. Med. Trop., Uberaba, v. 53, 
e20200167,

2020.

Disponível

em:

http://www.scielo.br/scielo.php?script=sci_arttext\&pid=S0037-

$86822020000101000 \& \operatorname{lng}=\mathrm{en} \& \mathrm{nrm}=\mathrm{iso}$

4. Vianna, Rafaella. Brasil tem 3.313 mortes e 49.492 casos de coronavírus, diz ministério. $\quad$ G1, 2020. Disponível em: https://g1.globo.com/bemestar/coronavirus/noticia/2020/04/23/brasil-tem-3313mortes-e-49492-casos-de-coronavirus-diz-ministerio.ghtml

5. Forte, Pablo Dias et al. O direito à renda básica no Brasil em tempos de Covid-19. Observatório COVID-19: $3 p \quad 2020 . \quad$ Disponível em: https://www.arca.fiocruz.br/handle/icict/40786

6. Cechinel, A; Fontana SAP.; Giustina K P D.; Pereira, AS.; Prado SS. Estudo/Análise Documental: Uma Revisão Teórica E Metodológica. Criar Educação UNESC, Criciúma, v. $5, \quad \mathrm{n}^{\circ} 1, \quad$ janeiro/Junho 2016. Disponível em: http://periodicos.unesc.net/criaredu/article/view/2446/2324

7. De Souza J, Kantorski, LP, Villar, MAL. Análise documental e observação participante na pesquisa em saúde mental. Revista Baiana de Enfermagem, Salvador, maio/ago. 2011 (online), 25 (2): 221-228. Disponível em: https://pdfs.semanticscholar.org/fd64/d17fda1279a5381965cb8769ac23c8bfcfda.pdf

8. Ministério da Saúde. Secretaria de Vigilância em Saúde. Departamento de Vigilância das Doenças Transmissíveis. Protocolo de tratamento de Influenza: 2017 [recurso 
eletrônico] / Ministério da Saúde, Secretaria de Vigilância em Saúde, Departamento de Vigilância das Doenças Transmissíveis. - Brasília: Ministério da Saúde, 2018. Disponível em: https://bvsms.saude.gov.br/bvs/publicacoes/protocolo_tratamento_influenza_2017.p df

9. Anvisa (Brasil). Nota técnica gvims/ggtes/anvisa $n^{\circ}$ 04/2020. Orientações para serviços de saúde: medidas de prevenção e controle que devem ser adotadas durante a assistência aos casos suspeitos ou confirmados de infecção pelo novo coronavírus $\begin{array}{llll}\text { (sars-cov-2). } \quad \text { - } & \text { 31.03.2020. } & \text { Disponível }\end{array}$ http://portal.anvisa.gov.br/documents/33852/271858/Nota+T\%C3\%A9cnica+n+042020+GVIMS-GGTES-ANVISA/ab598660-3de4-4f14-8e6f-b9341c196b28

10. Delfini PSS e Reis AO. A Articulação entre serviços públicos de saúde nos cuidados voltados à saúde mental infantojuvenil. Cad. Saúde Pública, Rio de Janeiro, 28(2):357-366, fev, 2012. Disponível em: http://www.scielo.br/pdf/csp/v28n2/14.pdf

11. Costa, FB. A Saúde Mental Em Meio À Pandemia Covid-19. Disponível em: http://www.saude.df.gov.br/wp-conteudo/uploads/2018/03/Nota-Informativa-ASa\%C3\%BAde-Mental-e-a-Pandemia-de-COVID19-poss\%C3\%ADveis-impactose-dicas-de-gerenciamento-para-a-popula\%C3\%A7\%C3\%A3o-geral.pdf 